\title{
Field-Dependent Specific Heat and Energy Gap in the $\mathrm{Yb}_{4} \mathrm{As}_{3}$ Compound
}

\author{
R. MATYSIAK* \\ Institute of Engineering and Computer Education, University of Zielona Góra \\ Prof. Z. Szafrana 4, 65-516 Zielona Góra, Poland
}

We report the low-temperature specific-heat simulation results and reanalyzed measurement data on polydomain $\mathrm{Yb}_{4} \mathrm{As}_{3}$ in magnetic fields $B=0$ and $6 \mathrm{~T}$. The data considered here are independent and supplement those considered earlier. A quantitative agreement has again been achieved between the magnetic experimental specific-heat data and the numerical results obtained by the quantum transfer-matrix simulation technique, yielding a new evidence in favour of the Heisenberg model of the antiferromagnetic spin $S=1 / 2$ chain with the value of the exchange integral $J / k_{\mathrm{B}}=-28 \mathrm{~K}$. The finite-size quantum transfer matrix approximants have been extrapolated exploiting the procedure developed previously. On the basis of the data in magnetic field and using the corresponding density-matrix renormalization group results, the energy-gap size $\Delta$ has been estimated for the applied magnetic field $B=6 \mathrm{~T}$, leading to the experimental verification in the extended region of the scaling law $\Delta \sim B^{2 / 3}$ following from the sine-Gordon model.

PACS numbers: 75.10.Jm, 75.40.Cx, 75.40.Mg, 71.55.Ak

\section{Introduction}

At high temperatures, $\mathrm{Yb}_{4} \mathrm{As}_{3}$ is a homogeneous intermediate valent (IV) metal with a valence ratio of $\mathrm{Yb}^{2+} / \mathrm{Yb}^{3+}=3: 1$. Three quarters of the $\mathrm{Yb}$ ions have filled $4 f$ shells with the valency $2+$ and one quarter with the valency $3+$ has one hole in the $f$ shell. The $\mathrm{Yb}$ ions reside statistically on four equivalent families of chains along the space diagonals of a cube. At low temperatures, the $\mathrm{Yb}^{3+}$ ions form a one-dimensional spin $S=1 / 2$ chain along the $\langle 111\rangle$ direction. The remaining $\mathrm{Yb}$ ions occupy nonmagnetic divalent states [1]. The $J=7 / 2$ ground-state multiplet splits into four doublets as a result of the crystal-field effect. Thus, the low-temperature dynamics is described by an effective $S=1 / 2$ spin chain with antiferromagnetic interactions and staggered field $[2,3]$. However, some effort was needed to model the specific heat results for $\mathrm{Yb}_{4} \mathrm{As}_{3}[4,5]$. Finally, it was achieved in [6], using the Hamiltonian

$$
\begin{aligned}
\boldsymbol{H} & =-J \sum_{i=1}^{N} \boldsymbol{S}_{i} \boldsymbol{S}_{i+1}-g_{\perp} \mu_{\mathrm{B}} B^{x} \sum_{i=1}^{N} S_{i}^{x} \\
& -g_{\perp} \mu_{\mathrm{B}} B_{s}^{y} \sum_{i=1}^{N}(-1)^{i} S_{i}^{y},
\end{aligned}
$$

where $B^{x}=B \cos (\theta), B_{s}^{y}=B \sin (\theta), B$ is the uniform external magnetic field perpendicular to the one-dimensional spin chain, $g_{\perp}=1.3$ and $g_{\|}=3.0[2]$, $\tan (\theta)=0.19[3]$ and the intrachain exchange coupling $J / k_{\mathrm{B}}=-28 \mathrm{~K}$.

In order to accomplish the task [6], the ingredients of the experimental specific heat data were carefully analyzed, yielding the new coupling constant $J / k_{\mathrm{B}}=-28 \mathrm{~K}$ and the new estimate of the lattice contribution in the

* e-mail: r.matysiak@eti.uz.zgora.pl form

$$
C_{\mathrm{ph}} / T=\alpha T^{2}+\beta T^{4}
$$

where $\alpha=1.11 \times 10^{-3} \mathrm{~J} /\left(\mathrm{mol} \mathrm{K}{ }^{4}\right)$ and $\beta=4.9 \times$ $10^{-6} \mathrm{~J} /\left(\operatorname{mol~K}{ }^{6}\right)$. Using our specific heat [6], the scaling of the energy gap was verified in the narrow region of fields $3.84 \leq B_{\text {eff }} \leq 6.95 \mathrm{~T}$. The effective field $B_{\text {eff }}$ is discussed in the subsequent part of the work.

The agreement [6] between the experimental specific heat data and those following from the model (1) as well as the support for the gap scaling are remarkably good. However, in order to confirm the reliability of the model, its parameters and the scaling law, new experimental data for $\mathrm{Yb}_{4} \mathrm{As}_{3}$ are of high interest. Such data, performed on different sample and experimental setup, are present in Ref. [7]. Here we select the representative cases $B=0$ and $B=6 \mathrm{~T}$. The latter is also attractive, because it corresponds to $B_{\text {eff }} \leq 3.2 \mathrm{~T}$ and may shift the lower bound of the range of the experimental verification of the gap scaling which is not trivial to achieve.

In order to calculate the field-dependent magnetic specific heat we assumed that $25 \%$ of the domains were oriented in parallel and $75 \%$ of domains were oriented perpendicular to the direction of the spin chain. The external magnetic field $B$ is applied in parallel to the chain direction and for $75 \%$ of domains the effective magnetic field $B_{\text {eff }}=B \sin (70)$ is assumed to be oriented in perpendicular to the direction of the chain. Finally, the numerical specific heat result is given by $C / T(B)=0.75 C_{\perp} / T\left(B_{\text {eff }}\right)+0.25 C_{\|} / T(B)$.

\section{Results and discussion}

The magnetic specific heat results for magnetic field $B=0 \mathrm{~T}$ and $B=6 \mathrm{~T}$ are presented in Fig. 1 . The full symbols represent numerical results. The open symbols represent the experimental data [7] collected for $B=6 \mathrm{~T}$ after subtraction of the phonon contribution (2). The 
results of the simulations are in good agreement with the experimental data in the whole range of temperatures both for the zero-field and the in-field specific heat, similar to that established before [6].

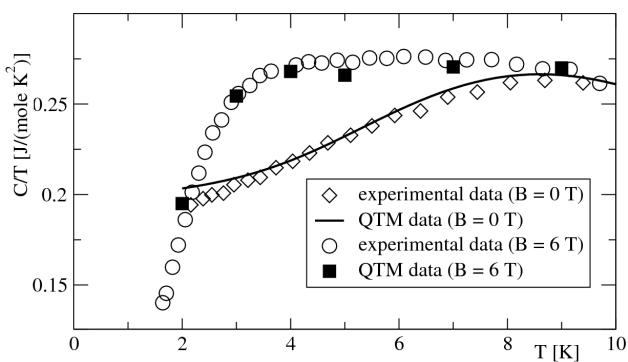

Fig. 1. Temperature dependences of the magnetic specific-heat data for $B=0$ and $6 \mathrm{~T}$. Open symbols correspond to the experimental data [7] after subtraction of the lattice contribution, while filled squares to the results of the simulations. The results of the QTM calculations in zero field are shown by continuous line.

Opening of the gap is an important feature of model (1). The size of the gap $\Delta(B)$ is related to the position of the maximum $T_{\max }(B)$ of the peaks in the temperature dependence of $C(B) / T[2]$. These positions depend, however, only on the value of the uniform field component $B^{x}$, whereas experimental data for the polydomain sample contain the component $C_{\|} / T$ which originates from the chains parallel to the field. This longitudinal component can be estimated numerically and subtracted from the experimental data, yielding the transverse part $C_{\perp} / T=C_{\exp } / T-C_{\|} / T$. The procedure to relate the position $T_{\max }$ of the maxima in our $C_{\perp}(B) / T$ with the energy gap is described in the previous paper [6] and is exploited here.

On the basis of experimental data published in [7] and results of the present simulations, needed to extract the part corresponding to the perpendicular field direction, the experimental value of $\Delta$ is estimated for the magnetic field of $B=6 \mathrm{~T}$. Figure 2 presents the gap dependence on the magnetic field obtained on the basis of inelastic neutron scattering (INS) measurements (full circles) and that obtained from the specific heat measurements which is plotted by full squares and an open square. The former are taken from [6] and the latter is calculated here. The value found in the present paper for $B=6 \mathrm{~T}$ fits to the linear behaviour calculated for the higher fields $B=8$, 12,16 and $19.5 \mathrm{~T}$ in $[6]$ and is significant. It extends the field region for the experimental verification of the gap scaling law.

\section{Concluding remarks}

The temperature dependence of the specific heat of $\mathrm{Yb}_{4} \mathrm{As}_{3}$ in magnetic fields $B=0$ and $6 \mathrm{~T}$ and the numerical results, based on the Heisenberg model (1) with staggered field, are successfully compared. Good agreement obtained over the whole range of temperature for

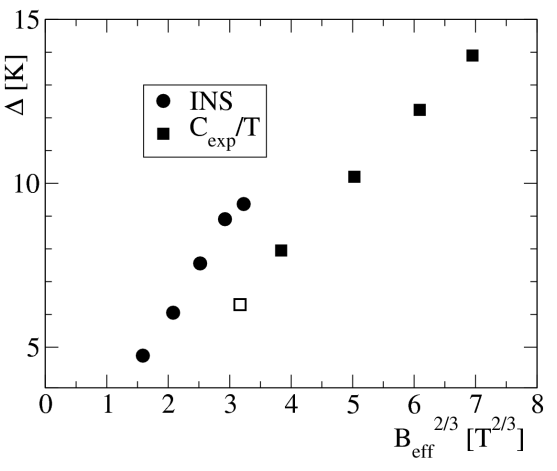

Fig. 2. The energy gap values as a function of the magnetic field. The experimental results obtained by INS are marked with full circles. The energy gap obtained from the analysis of the specific heat data are marked by full squares and an open square for the previous [6] and present calculations, respectively.

the same values of parameters enhances reliability of the model (1) as far as the magnetic properties of $\mathrm{Yb}_{4} \mathrm{As}_{3}$ are concerned.

Moreover, on the basis of the results obtained from the model (1), the energy gap was estimated from experiment performed on a different sample for $B=6 \mathrm{~T}$. The estimate fits to the linear behaviour in $B^{2 / 3}$ found before [6] for magnetic fields $B>6 \mathrm{~T}$ and confirms experimentally the scaling in a wider range of magnetic fields.

\section{Acknowledgments}

I would like to thank Prof. G. Kamieniarz for many valuable discussions. This work was supported by the Polish Ministry of Science and High Education grant N202 230137. Computations were partially performed on PCSN supercomputing platforms.

\section{References}

[1] M. Köppen, M. Lang, R. Helfrich, F. Steglich, P. Thalmeier, B. Schmidt, B. Wand, D. Pankert, H. Benner, H. Aoki, A. Ochiai, Phys. Rev. Lett. 82, 4548 (1999).

[2] M. Kohgi, K. Iwasa, J.-M. Mignot, B. Fak, P. Gegenwart, M. Lang, A. Ochiai, H. Aoki, T. Suzuki, Phys. Rev. Lett. 86, 2439 (2001).

[3] N. Shibata, K. Ueda, J. Phys. Soc. Jpn. 70, 3690 (2001).

[4] G. Kamieniarz, R. Matysiak, P. Gegenwart, H. Aoki, A. Ochiai, J. Magn. Magn. Mater. 290-291, 353 (2005).

[5] G. Kamieniarz, R. Matysiak, P. Gegenwart, H. Aoki, A. Ochiai, Inorg. Chim. Acta 360, 3955 (2007).

[6] R. Matysiak, G. Kamieniarz, P. Gegenwart, A. Ochiai, Phys. Rev. B 79, 224413 (2009).

[7] F. Steglich, M. Köppen, P. Gegenwart, T. Cichorek, B. Wand, M. Lang, P. Thalmeier, B. Schmidt, H. Aoki, A. Ochiai, Acta Phys. Pol. A 97, 91 (2000). 